\title{
Development OF Haptic InTERface to Simulate Vocal Fold SURGERY
}

\author{
Arlindo N. Mont agnoli ${ }^{1}$, Lucas R. B. Souza ${ }^{1}$, Daniel Diegues ${ }^{1}$, Giuseppe A. Cirino ${ }^{1}$, José B. Rubert ${ }^{1}$,
} Regina A. Pimenta ${ }^{2}$, Paula B. Baravieira ${ }^{2}$, Monike Tsutsumi², Maria E. Dajer ${ }^{3}$, Domingos H. Tsuji ${ }^{3}$.

\author{
1. Department of Electrical Engineering, Federal University of São Carlos - UFSCar \\ São Carlos, Brazil \\ E-mail: arlindo@ufscar.br
}

\section{Department of Bioengineering, University of São Paulo-EESC-USP São Carlos, Brazil \\ E-mail: moniketsutsumi@usp.br}

\author{
3. Department of Otorhinolaryngology, University of São Paulo - USP \\ São Paulo, Brazil. \\ E-mail: dtsujieusp.br
}

\begin{abstract}
The presence of robots in surgery rooms made possible the development of the so-called minimally invasive surgeries (MIS), and also allowed the emergence of robotic telesurgery. The dynamic control of these systems should be designed aiming at producing forces and complex movements such as incisions, dissections, and sutures. The dynamic controls are present not only in handlers but also in actuators and sensors that simulate the presence of organs, providing the tactile feedback of the different structures. These devices are called haptic interfaces. They are used in the medicine field on many types of virtual surgeries. Computational methods were developed and used to simulate the movements of the vocal folds in three dimensions, using the anisotropic body-cover concept to define the structure. The project carried out consists in a haptic interface able to reproduce the sense of touch in small structures as vocal folds.
\end{abstract}

Keywords_ Haptic Interface, Virtual Surgery, Larynx, Vocal Folds.

\section{Introduction}

It's called a haptic interface the electro-mechanical computer that allows the user to interact with an image in virtual reality through the sense of touch. These devices consist of an articulated mechanical structure where a dedicated electronic provides the servo motors control capable of simulating the touch feeling of the resistant forces inherent to the virtual structural stiffness against the realized movements. The haptic interface simulating a video-laparoscopic exam while manipulating virtual objects and organs is showed in Figure 1 (Khatounian, Moreau, Louis, et al. 2006b).
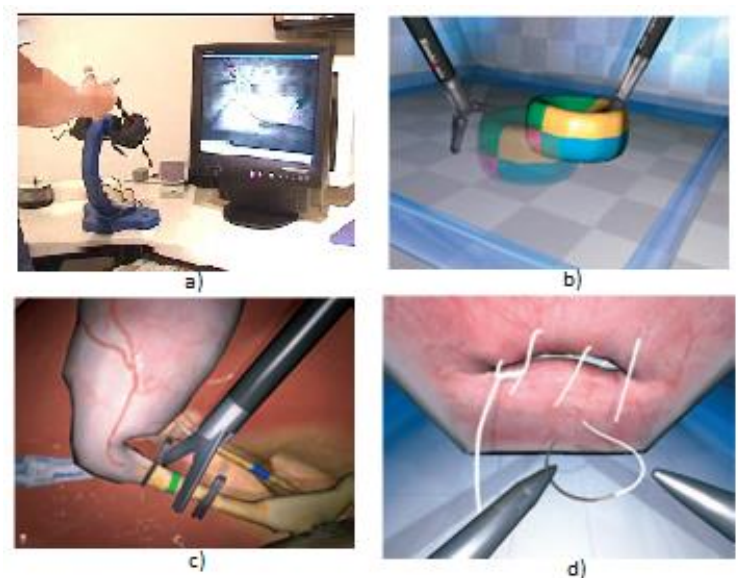

Figure 1: a) Haptic interface simulating a video-laparoscopy, b) manipulating virtual objects, c) dissection d) suture virtual organs (Khatounian, Moreau, Louis, et al. 2006a).
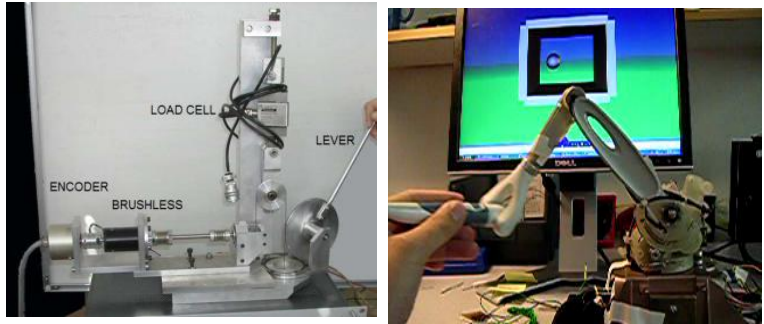

Figure 2: Development of our conceptual version and a commercial haptic interface.

Figure 2 a shows a developed stage of haptic interface and figure 2b.a commercial one. Figure 3 shows an example of real use application.

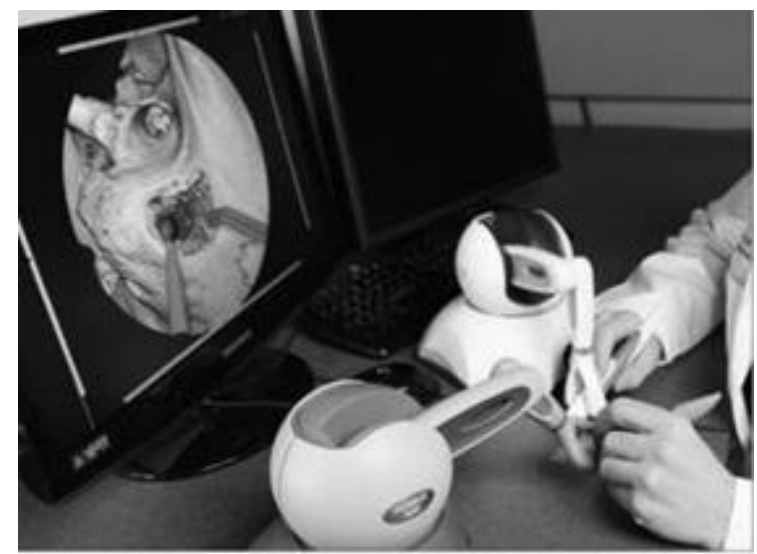

Figure 3:Finished haptic interface simulating a bone surgery, (Nogueira Jr. \& Cruz 2010). 
For the numerical simulation of the human voice production we need a method that involves a physiological model, which includes the neural control of the lungs, trachea, larynx, vocal tract and radiation, making it too complex and difficult to solve. The model of one mass of (J. Flanagan \& Landgraf 1968) was the first model to simulate the selfoscillation of the vocal folds. An improved model developed by Ishizaka and Flanagan gives closer match with results of the real movements of the vocal cords results, and therefore is widely accepted. It is also usually a precursor model to more elaborate methods. This model considers the glottis as two masses interconnected by a spring vibrating on two springs and two dampers shown in Figure 4 (Ishizaka \& J. L. Flanagan 1972).
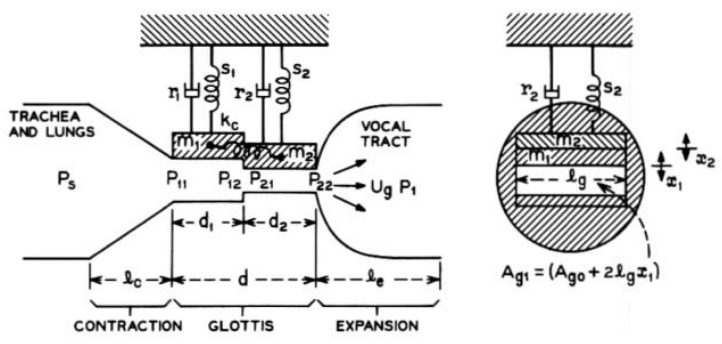

Figure 4:Schematic diagram of the two mass approximation of the vocal folds (Ishizaka \& J. L. Flanagan 1972).

The spring-mass models provide us information about the physical behavior of the vocal folds and the airflow in the glottis in a simulated system. The dynamics of the vocal folds has been extensively studied for several decades, having developed a number of models of the vocal folds. The models most often cited as precedents of the methods currently used include the model of a body and two masses, the model of multiple masses, the continuous model, the model of four parameters and body-cover model, ( $\mathrm{J}$. L. Flanagan \& Cherry 1969)(Ishizaka \& J. L. Flanagan 1972)(Hirano \& Kakita 1985)(Zhang \& Jiang 2004). The model presented in this work (?) allows two masses split the vocal into two parts enabling the analysis of the phase difference observed in the movement of the vocal folds. Each part consists of an oscillator mass-spring-damper, in which the spring is equivalent to the elastic property of the vocal and the damper represent an effect of dissipative forces, like friction and viscosity. There is also an interaction between the two masses, represented by the coupling, which is the restoring force balance that occurs between two masses when they are displaced with respect to their respective positions. This model enables more realistic simulations of glottal properties with relatively less computational effort. The concept of body-cover of (Hirano \& Kakita 1985 ) is usually used to describe the layered structure of the vocal folds. This model suggests that the vocal cords can be divided into two tissue layers with different mechanical properties. The layer of the body consists of muscle fibers stuck firmly in the collagen fibers of the vocal ligament. The cover layer consists of flexible tissue that surrounds the body layer. The movement of the cover layer can be observed as a wave surface originating from the bottom to the top of the vocal folds, spreading in the lateral and vertical directions.

\section{Methodology}

The algorithms for processing and visualization of images were developed using the Visualization Toolkit package (VTK), all open source. The routines for the solution of dynamic meshes were developed using C++ language standard, as MeshLab and Siemens NX 8.0 were also used as complementary software. Computer Tomography (CT) was performed using the Siemens Emotion CT scanner Somation and for high-speed laryngoscopy was used Endocam 5262 from Richard Wolf.

The geometry is used to represent object shape, and physics imposes restrictions on how the shape may vary over space that generally deforms under the laws of Newton mechanics. Deformable model allow an initial shape converges to a new geometric representation, the deformation that a model can undergo is not arbitrary, but should satisfy some problem specific constraints. The physical interpretation of deformable models is seen as an elastic body that naturally responds to external forces, imposing restrictions on its deformation (Alipour et al. 2000)(Kass et al. 1988). In the deformable threedimensional model employed in this work, a vocal folds frame is composed of a set of nodes forming a frame by springs and dampers connected in parallel. Figure 5 shows schematically this arrengment. The positions vector of $(i, j, k)$-th mesh point $\boldsymbol{p}_{i, j, k}=[x, y$, $z]^{T}$, with $i=1, \ldots, L ; j=1, \ldots, M$; and $k=1, \ldots, N$, where $L, M$, and $N$, are the amount of points in three dimension, used to describe translation, rotation and deformation of the object.

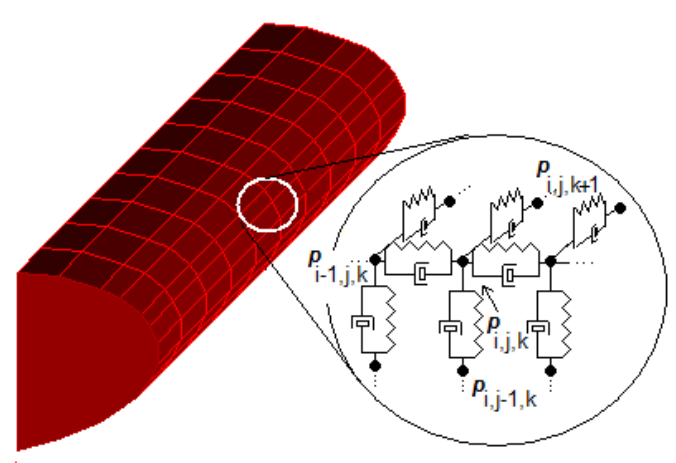

Figure 5: Details of vocal fold's mesh used in this work.

The Newtonian equation: $F=k a+b a^{\prime}+m a^{\prime \prime}$ describes the dynamic equilibrium of each vertex and their differential equations were solved explicitly with central differences time integration. This meth- 
od was chosen due to its speed and easeness of modifications, such as the ability to adapt the mesh to provide a better performance for real-time response required by haptic interfaces (Zhou et al. 2012)(A.N. Montagnoli \& Fonseca 2005). To solve the equation of the movement, the algorithm of central differences is used assuming itself known the displacement $a$ in the instant of time $t^{n}$, the velocity $\dot{a}$ in the instant of time $t^{n-1 / 2}$, internal forces $f_{i}(a)$ and external forces $f_{e}$, as presented in the Equations (1-4) to follow:

$$
\begin{aligned}
& \ddot{a}=M^{-1}\left[f_{e}-f_{i}(a)\right] \\
& \dot{a}^{n+1 / 2}=\dot{a}^{n-1 / 2}+\ddot{a}^{n} \Delta t^{n} \\
& a^{n+1}=a^{n}+\dot{a}^{n+1 / 2} \Delta t^{n+1 / 2} \\
& \Delta t^{n+1 / 2}=\frac{1}{2}\left(\Delta t^{n}+\Delta t^{n+1}\right)
\end{aligned}
$$

Where $\Delta t^{n}$ and $\Delta t^{n+1}$ are successive time intervals respectively between $t^{n-1}$ to $t^{n}$, and $t^{n}$ to $t^{n+1}$.

A brushless motor was used because of its excellent dynamic response, low noise, friction and inertia, and powered by its respective driver. A control system with a PID controller was used and an incremental encoder connected to a PID controller closes the control loop. This controller was implemented in a dedicated microcontroller ARM (LPC1769) that manipulates the input parameters from the computational model by means of a voltage and current in the motor. The user moves the lever and has the feel of acting on a real object since the emulation takes into account a mass-spring-damper system where the parameters are changed dynamically with the virtual contact on the monitor screen.

The personal computer generates a rendered object from a deformable model and shows to the user a three-dimensional image on the video monitor. It communicates with the haptic interface through USB communication port. The haptic interface sends the information from the incremental encoder that contains the coordinate from lever position. The computer returns to the haptic interface how much torque the PID controller should keep the lever in a certain position. This interaction anable the user of interface to have the feeling of touching objects with different hardness. A flowchart of the basic interface is shown in Figure 6 below.

The developed software receives information of the coordinates of the interface and compares with the coordinates of the object. If interface senses any contact the deformable model algorithm is executed, returning to the interface the force value that the motor should remain in that position, causing the user interface to transmit a genuine sense of touch. The haptic interface was developed with a single degree of freedom, using an electrical motor in order to simplify the model proposed in Figure 5,

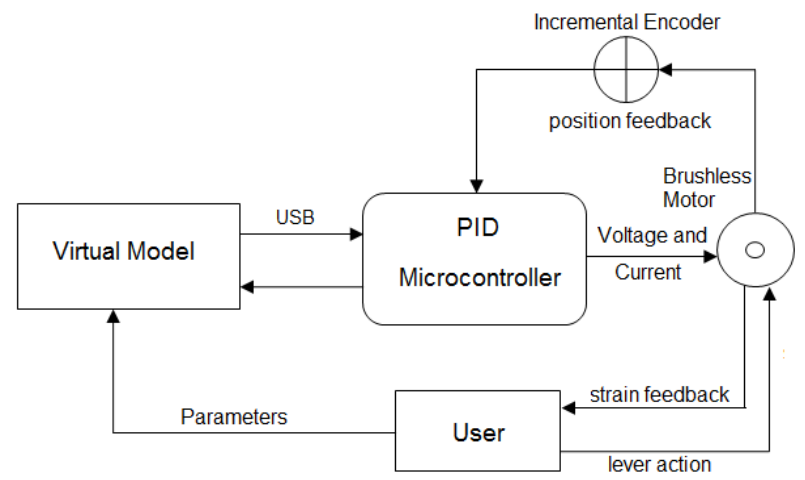

Figure 6: Block diagram of developed haptic interface hardware.

It is a mechanical structure with a single degree of freedom, similar to proposed by (Khatounian, Moreau, Monmasson, et al. 2006), and was assembled replacing the drive belt system by a pinion and gear. Figure 7 shows schematically the mechanical model proposed for the study of haptic interface with one degree of freedom. This structure was chosen to allow initial validation study and enable to expand to haptic interfaces with higher degrees of freedom, for example, an interface with three degrees of freedom allow the rotation of the base and two joints in the arm.

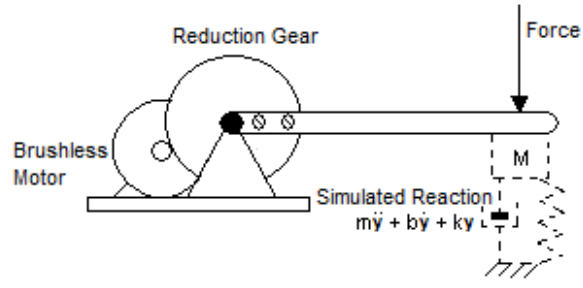

Figure 7: Mechanical model proposed for the study of haptic interface with one degree of freedom.

\section{Results}

The model for carrying out tests for haptic interface was done by a deformable bar created from interactions of a spring mass system, being touched by a spherical object, showed in Figure 9
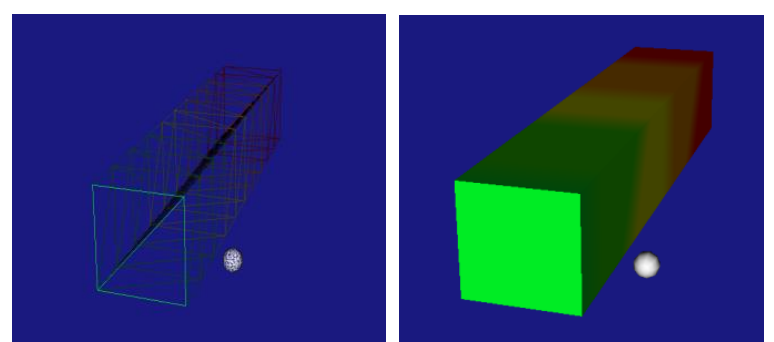

Figure 9: Simple cantilever beam to validate the virtual deformable model. a) mesh frame model, b) rendered model. 


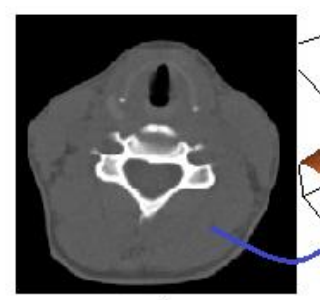

a)

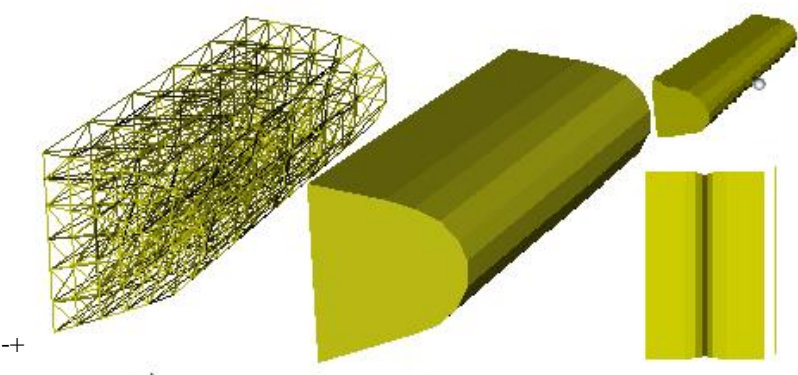

e)

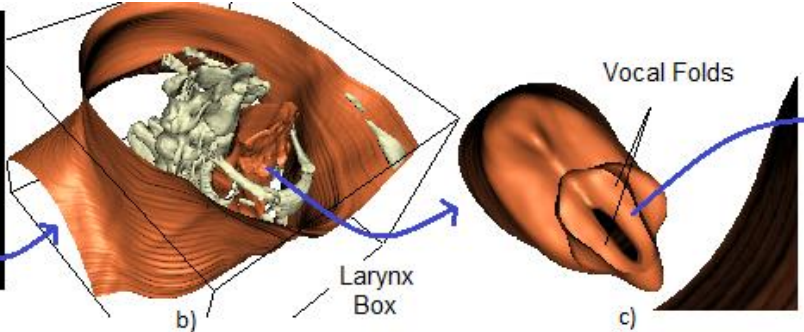

c)

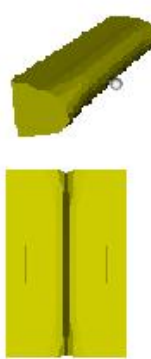

f)

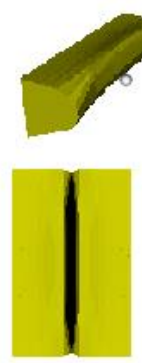

g)
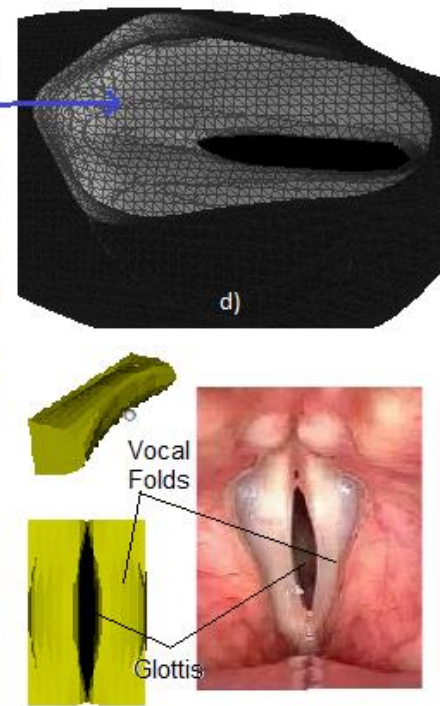

h)

Figure 8: a) CT image, b) 3D reconstruction, c) segmentation of the larynx, d) obtaining the mesh e) mesh of the vocal fold, f) vocal fold rendered, $\mathrm{g}$ ) sequence of forced opening movements, h) vocal folds view by laryngoscope.

All stages of development of the deformable model of the vocal folds can be seen in Figure 8. The entire process starts from the $3 \mathrm{D}$ reconstruction of tomography images followed by segmentation of the vocal fold. Then the developed mesh deformable model was used to simulate the movement provided by the interaction with the haptic interface, (Arlindo $\mathrm{N}$ Montagnoli et al. 2010).

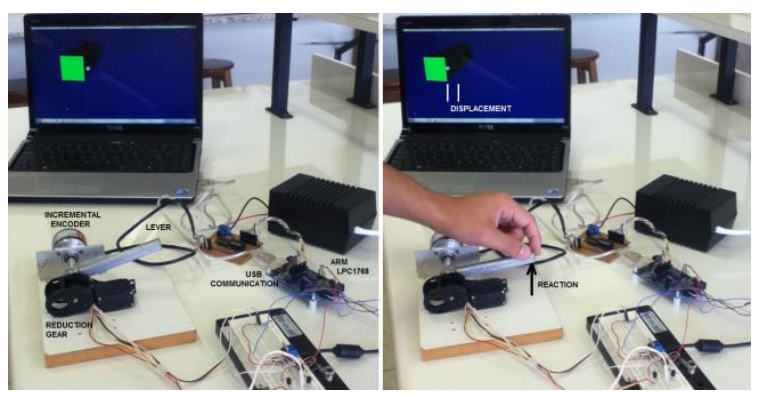

Figure 10: Hapitic implementation, with incremental encoder and ARM LPC1768.

A qualitative comparison between cantilever and vocal fold was made applying an instantaneous force on opposite side of fixed points. The vocal fold time response was very slow. In this test we need to reduce the time step for an impracticable value for a real-time response, due to the compromise between the number of connections, and the time constant for the model algorithm converges. Figure 10 shows a photography of the haptic interface prototype.

\section{Conclusions}

The use of interfaces that allows the user to have a feeling of interacting with a virtual reality is proving increasingly sought and searched. One can see these characteristics in most equipment being used for visualizations in three dimensions, and gaming devices which present a more realistic scenario, among other examples. Thus, the search for sensations of touch in a virtual reality also is being sought and is already being used in several professional simulators such as flight, race, space and surgery. Therefore the project developed here shows very promising and applicable in different fields.. The final result was satisfactory for presenting virtual model visualization with a USB communication between software and hardware and with satisfactory speed. The satisfactory term is used in this context to express the touch sensation that the human being perceives to be hoodwinked by haptic interface. It was noticed that the human being is potentially susceptible to mistake, and it was noted that only with visual feedback even with the interface turned off he has a sensation of strength, but were not made measurements of the real value that the engine applies on the lever. The sense of touch obtained at the end of the project brought the user a sense of touch reliable and realistic, as expected. It is concluded that the greatest difficulty about real-time response of the haptic interface was due to the processing time of the mesh of virtual model. Even with an explicit solution for body-cover model of the vocal folds, the answer geometrically and mechanically were similar to vocal behavior, 
however with dynamic too slow for real-time simulation of vocal folds and it was very realistic for a cantilever beam. Some alternatives as parallel processing and adaptive meshes are being analyzed for future implementation.

\section{Acknowledges}

Thanks to financial support from FAPESP: 2011/17858-2, CNPq: 152956/2011-5, and CNPq: $138061 / 2011-4$.

\section{References}

Alipour, F., Berry, D. a \& Titze, I.R., 2000. A finiteelement model of vocal-fold vibration. The Journal of the Acoustical Society of America, 108(6), pp.3003-12. DOI: $10.1121 / 1.1324678$

Flanagan, J. \& Landgraf, L., 1968. Self-oscillating source for vocal-tract synthesizers. IEEE Transactions on Audio and Electroacoustics, 16(1), pp.57-64. DOI: 10.1109/TAU.1968.1161949

Flanagan, J.L. \& Cherry, L., 1969. Excitation of vocal-tract synthesizers. The Journal of the Acoustical Society of America, 45(3), pp.764-9. DOI: 10.1121/1.1911461

Hirano, M. \& Kakita, Y., 1985. Cover-body theory of vocal cord vibration. In San Diego: R. G. Daniloff College Hill Press, pp. 1-46.

Ishizaka, K. \& Flanagan, J.L., 1972. Synthesis of voiced sounds from a two-mass model of the vocal cords. Bell System Technical Journal, 51(6), pp.1233-1268. DOI: 10.1002/j.1538-7305.1972.tb02651.x

Kass, M., Witkin, A. \& Terzopoulos, D., 1988. Snakes: Active contour models. International Journal of Computer Vision, pp.321-331. DOI: 10.1007/BF00133570

Khatounian, F., Moreau, S., Louis, J., et al., 2006. Modeling and simulation of a hybrid dynamic system used in haptic interfaces. Mathematics and Computers in Simulation, 71(4-6), pp.270281. DOI: 10.1016/j.matcom.2006.02.021

Khatounian, F., Moreau, S., Monmasson, E., et al., 2006. Speed Estimation Improvement After Decreasing the Encoder Resolution for a Haptic Interface. 2006 IEEE International Symposium on Industrial Electronics, (1), pp.341-346. DOI: 10.1109/ISIE.2006.295617

Montagnoli, A.N. \& Fonseca, E.S., 2005. Larynx Virtual Surgery. In Seventh IEEE International Symposium on Multimedia (ISM'05). IEEE, pp. 790-795.
Montagnoli, Arlindo N et al., 2010. Computational Simulations in Mass-Spring Dynamic System to the Development of Vocal Folds Tissues Models. In Brazilian Conference on Dynamics, Control and their Applications. pp. 1-6.

Nogueira Jr., J.F. \& Cruz, D.N., 2010. Real models and virtual simulatos in otolaryngology: review of literature. Brazilian Journal of Otorhinolaryngology, 76(1), pp.129-135. DOI: 10.1590/S1808-86942010000100021

Zhang, Y. \& Jiang, J.J., 2004. Chaotic vibrations of a vocal fold model with a unilateral polyp. The Journal of the Acoustical Society of America, 115(3), pp.1266-9. DOI: $10.1121 / 1.1648974$

Zhou, M. et al., 2012. Effect of haptic feedback in laparoscopic surgery skill acquisition. Surgical endoscopy, 26(4), pp.1128-34. DOI: 10.1007/s00464-011-2011-8 